\title{
DHAGE ITERATION METHOD FOR APPROXIMATING THE POSITIVE SOLUTIONS OF IVPS FOR NONLINEAR FIRST ORDER QUADRATIC NEUTRAL FUNCTIONAL DIFFERENTIAL EQUATIONS WITH DELAY AND MAXIMA
}

\author{
Bapurao C. Dhage ${ }^{1}$, Shyam B. Dhage ${ }^{2}$, John R. Graef ${ }^{3} \S$ \\ ${ }^{1,2}$ Kasubai, Gurukul Colony \\ Ahmedpur - 413 515, Dist: Latur, Maharashtra, INDIA \\ ${ }^{3}$ Department of Mathematics \\ University of Tennessee at Chattanooga \\ Chattanooga, TN 37403, USA
}

\begin{abstract}
In this paper, the authors prove an existence and approximation theorem for positive solutions to a nonlinear first order quadratic hybrid neutral functional differential equations with delay and maxima under mixed geometric, algebraic, and topological conditions. They employ the Dhage iteration method embodied in a hybrid fixed point principle of Dhage (2014) involving the product of two operators in a partially ordered Banach algebra.

A numerical example is also provided to indicate the applicability of the abstract result to a concrete problem.
\end{abstract}

AMS Subject Classification: 34A12, 34A45, 47H07, 47H10

Key Words: quadratic neutral functional differential equation, hybrid fixed point principle, Dhage iteration method, existence and approximation theorem, equations with maxima

Received: November 15, 2017

(c) 2018 Academic Publications

$\S_{\text {Correspondence author }}$ 


\section{Statement of the Problem}

Given real numbers $r>0$ and $T>0$, we consider the closed and bounded intervals $I_{0}=[-r, 0]$ and $I=[0, T]$ of the real line $\mathbb{R}$ and let $J=[-r, T]$. By $\mathcal{C}=C\left(I_{0}, \mathbb{R}\right)$ we denote the class of continuous real-valued functions defined on $I_{0}$. We equip the vector space $\mathcal{C}$ with he norm $\|\cdot\|_{\mathcal{C}}$ defined by

$$
\|x\|_{\mathcal{C}}=\sup _{-r \leq \theta \leq 0}|x(\theta)|
$$

Clearly, $\mathcal{C}$ is a Banach space with this supremum norm and it is sometimes called the history space of the functional differential equation in question.

For any continuous function $x: J \rightarrow \mathbb{R}$ and for any $t \in I$, we denote by $x_{t}$ the element of the space $\mathcal{C}$ defined by

$$
x_{t}(\theta)=x(t+\theta),-r \leq \theta \leq 0 .
$$

Consider the nonlinear first order quadratic hybrid functional differential equation (in short QHFDE) with a delay and maxima,

$$
\left.\begin{array}{c}
\left(\frac{x(t)}{f(t, x(t), X(t))}\right)^{\prime}+\lambda\left(\frac{x(t)}{f(t, x(t), X(t))}\right)=g\left(t, x(t), x_{t}\right), t \in I, \\
x_{0}=\phi \in \mathcal{C}
\end{array}\right\}
$$

where $\lambda \in \mathbb{R}, \lambda>0, f: I \times \mathbb{R} \times \mathbb{R} \rightarrow \mathbb{R} \backslash\{0\}$ and $g: I \times \mathbb{R} \times \mathcal{C} \rightarrow \mathbb{R}$ are continuous functions with $X(t)=\max _{0 \leq \xi \leq t} x(\xi)$.

By a solution of the QHFDE (3) we mean a function $x$ in the space $C(J, \mathbb{R})$ of continuous real-valued functions defined on $J$ that satisfies (3).

The QHFDE (3) contains two different delay arguments, one is in the function $f$ in the form of the maxima function and the other is in the function $g$. Thus, this problem is new to the literature on functional differential equations. Differential equations with maxima occur in automatic regulated control systems whereas differential equations with past history occur in several natural and physical phenomena. The details of these facts are given in Hale [21], Myshkis [25], Magomedov [23], and the references therein. Differential equations with delay and maxima help to describe some mixed dynamic systems involving past history and the maximum range.

The QHFDE (3) is general in the sense that it includes some important classes of functional differential equations. If $f(t, x, y) \equiv 1$, then the QHFDE 
(3) reduces to the following FDE with a delay,

$$
\left.\begin{array}{c}
x^{\prime}(t)+\lambda x(t)=g\left(t, x(t), x_{t}\right), t \in I, \\
x_{0}=\phi
\end{array}\right\}
$$

and if $f(t, x, y)=f(t, x)$, then reduces to the quadratic FDE with delay,

$$
\left.\begin{array}{c}
\left(\frac{x(t)}{f(t, x(t))}\right)^{\prime}+\lambda\left(\frac{x(t)}{f(t, x(t))}\right)=g\left(t, x(t), x_{t}\right), t \in I, \\
x_{0}=\phi \in \mathcal{C} .
\end{array}\right\}
$$

Nonlinear functional differential equations have been studied in the literature for a long time via functional analytic methods; see Hale [21] and references therein. Similarly, quadratic functional differential and integral equations have also been studied for a long time; however the study gained momentum after the development of hybrid fixed point theorems in a Banach algebra due to Dhage [3]. But the study of FDEs via the Dhage iteration principle is relatively new to the literature. Very recently, a few results in this direction were obtained by Dhage [11] and a special class of FDEs was discussed in Dhage $[9,10]$ and Dhage and Dhage $[16,17]$. In this paper, we obtain existence and approximation results for the QHFDE (3) via the Dhage iteration method and develop an algorithm for the approximate or numerical solution. The FDE (4) and the QHFDE (5) are also new to existence and approximation via the Dhage iteration method. Therefore, the results of this paper includes the existence and approximation theorems for other functional differential equations as special cases which are also new to the literature. In the following section we give some preliminaries and auxiliary results that will be needed in the proof of the main results in Section 3.

\section{Auxiliary Results}

Unless otherwise mentioned, throughout this paper we let $E$ denote a partially ordered real normed linear space with an order relation $\preceq$ and norm $\|\cdot\|$ in which the addition and the scalar multiplication by positive real numbers are preserved by $\preceq$. Details on partially ordered normed linear space appear in Dhage [5], Heikkilä and Lakshmikantham [22], and the references therein.

Two elements $x$ and $y$ in $E$ are said to be comparable if either the relation $x \preceq y$ or $y \preceq x$ holds. A non-empty subset $C$ of $E$ is called a chain or totally 
ordered if all the elements of $C$ are comparable. The space $E$ is said to be regular if for any nondecreasing (resp. nonincreasing) sequence $\left\{x_{n}\right\}$ in $E$ such that $x_{n} \rightarrow x^{*}$ as $n \rightarrow \infty$, then $x_{n} \preceq x^{*}$ (resp. $x_{n} \succeq x^{*}$ ) for all $n \in \mathbb{N}$. Conditions guaranteeing the regularity of $E$ may be found in Heikkilä and Lakshmikantham $[22]$ and the references therein.

We need the following definitions (see Dhage $[5,6,7]$ and the references therein) in what follows.

Definition 1. A mapping $\mathcal{T}: E \rightarrow E$ is called isotone or monotone nondecreasing if it preserves the order relation $\preceq$, that is, if $x \preceq y$ implies $\mathcal{T} x \preceq \mathcal{T} y$ for all $x, y \in E$. Similarly, $\mathcal{T}$ is called monotone nonincreasing if $x \preceq y$ implies $\mathcal{T} x \succeq \mathcal{T} y$ for all $x, y \in E$. Finally, $\mathcal{T}$ is called monotonic or simply monotone if it is either monotone nondecreasing or monotone nonincreasing on $E$.

Definition 2. A mapping $\mathcal{T}: E \rightarrow E$ is called partially continuous at a point $a \in E$ if for $\epsilon>0$ there exists $\delta>0$ such that $\|\mathcal{T} x-\mathcal{T} a\|<\epsilon$ whenever $x$ is comparable to $a$ and $\|x-a\|<\delta$. The mapping $\mathcal{T}$ called partially continuous on $E$ if it is partially continuous at every point of $E$.

It is clear that if $\mathcal{T}$ is partially continuous on $E$, then it is continuous on every chain $C$ contained in $E$.

Definition 3. A non-empty subset $S$ of the partially ordered Banach space $E$ is called partially bounded if every chain $C$ in $S$ is bounded. An operator $\mathcal{T}$ on a partially normed linear space $E$ into itself is called partially bounded if $\mathcal{T}(E)$ is a partially bounded subset of $E$, and it is called uniformly partially bounded if all chains $C$ in $\mathcal{T}(E)$ are bounded by a unique constant.

Definition 4. A non-empty subset $S$ of the partially ordered Banach space $E$ is called partially compact if every chain $C$ in $S$ is a compact subset of $E$. A mapping $\mathcal{T}: E \rightarrow E$ is called partially compact if every chain $C$ in $\mathcal{T}(E)$ is a partially relatively compact subset of $E$, and $\mathcal{T}$ is called uniformly partially compact if $\mathcal{T}$ is a uniformly partially bounded and partially compact operator on $E$. An operator $\mathcal{T}$ is called partially totally bounded if for any bounded subset $S$ of $E, \mathcal{T}(S)$ is a partially relatively compact subset of $E$. If $\mathcal{T}$ is partially continuous and partially totally bounded, then we say it is partially completely continuous on $E$. 
Remark 5. Suppose that $\mathcal{T}$ is a nondecreasing operator on $E$ into itself. Then $\mathcal{T}$ is partially bounded or partially compact if $\mathcal{T}(C)$ is a bounded or compact subset of $E$ for each chain $C$ in $E$.

Definition 6. The order relation $\preceq$ and the metric $d$ on a non-empty set $E$ are said to be $\mathcal{D}$-compatible if $\left\{x_{n}\right\}_{n \in \mathbb{N}}$ is a monotone (monotone nondecreasing or monotone nonincreasing) sequence in $E$ and if a subsequence $\left\{x_{n_{k}}\right\}_{n \in \mathbb{N}}$ of $\left\{x_{n}\right\}_{n \in \mathbb{N}}$ converges to $x^{*}$ implies that the original sequence $\left\{x_{n}\right\}_{n \in \mathbb{N}}$ converges to $x^{*}$. Similarly, given a partially ordered normed linear space $(E, \preceq,\|\cdot\|)$, the order relation $\preceq$ and the norm $\|\cdot\|$ are said to be $\mathcal{D}$-compatible if $\preceq$ and the metric $d$ defined by the norm $\|\cdot\|$ are $\mathcal{D}$-compatible. A subset $S$ of $E$ is called Janhavi if the order relation $\preceq$ and the metric $d$ or the norm $\|\cdot\|$ are $\mathcal{D}$-compatible in it. In particular, if $S=E$, then $E$ is called a Janhavi metric or Janhavi Banach space.

Clearly, the set $\mathbb{R}$ of real numbers with the usual order relation $\leq$ and the norm defined by the absolute value function $|\cdot|$ has this property. Similarly, the finite dimensional Euclidean space $\mathbb{R}^{n}$ with the usual componentwise order relation and the standard norm possesses the $\mathcal{D}$-compatibility property. In general, every finite dimensional Banach space with a standard norm and an order relation is a Janhavi Banach space.

Definition 7. A upper semi-continuous and monotone nondecreasing function $\psi: \mathbb{R}_{+} \rightarrow \mathbb{R}_{+}$is called a $\mathcal{D}$-function provided $\psi(r)=0$ iff $r=0$. Let $(E, \preceq,\|\cdot\|)$ be a partially ordered normed linear space. A mapping $\mathcal{T}: E \rightarrow E$ is called partially nonlinear $\mathcal{D}$-Lipschitz if there exists a $\mathcal{D}$-function $\psi$ : $\mathbb{R}_{+} \rightarrow \mathbb{R}_{+}$such that

$$
\|\mathcal{T} x-\mathcal{T} y\| \leq \psi(\|x-y\|)
$$

for all comparable elements $x, y \in E$. If $\psi(r)=k r, k>0$, then $\mathcal{T}$ is called partially Lipschitz with a Lipschitz constant $k$.

Let $(E, \leq,\|\cdot\|)$ be a partially ordered normed linear algebra. Set

$$
\mathcal{K}=\{x \in E \mid x \geq \theta, \text { where } \theta \text { is the zero element of } E\},
$$

which is a closed and convex subset of $E$. The elements of $\mathcal{K}$ are called the positive vectors of the normed linear algebra $E$. The set $\mathcal{K}$ is called positive in view of the fact that it satisfies the relation " $u \cdot v \in \mathcal{K}$ whenever $u, v \in \mathcal{K}$." The next lemma follows immediately from the definition of the set $\mathcal{K}$ and is often used in the applications of hybrid fixed point theory in Banach algebras. 
Lemma 8. (Dhage [5]) If the elements $u_{1}, u_{2}, v_{1}, v_{2} \in \mathcal{K}$ are such that $u_{1} \preceq v_{1}$ and $u_{2} \preceq v_{2}$, then $u_{1} u_{2} \preceq v_{1} v_{2}$.

Definition 9. An operator $\mathcal{T}: E \rightarrow E$ is said to be positive if the range $R(\mathcal{T})$ of $\mathcal{T}$ satisfies $R(\mathcal{T}) \subseteq \mathcal{K}$.

The essential idea of the Dhage iteration principle may be described as "the monotonic convergence of the sequence of successive approximations to the solutions of a nonlinear equation beginning with a lower or an upper solution of the equation as its initial or first approximation." It is a very powerful tool in existence theory in nonlinear analysis. The procedure involved in the application of the Dhage iteration principle to nonlinear equations is called the "Dhage iteration method." It is clear that the Dhage iteration method is different for different nonlinear problems and is also different from the usual Picard successive approximation method. The Dhage iteration method embodied in the following applicable hybrid fixed point theorems of Dhage [6] is used as the key tool for our work contained in this paper. A few other hybrid fixed point theorems involving the Dhage iteration method may be found in Dhage $[6,7,8]$.

Theorem 10. (Dhage [7]) Let $(E, \preceq,\|\cdot\|)$ be a regular partially ordered complete normed linear algebra such that every compact chain $C$ of $E$ is Janhavi. Let $\mathcal{A}, \mathcal{B}: E \rightarrow \mathcal{K}$ be two nondecreasing positive operators such that:

(a) $\mathcal{A}$ is partially bounded and nonlinear partial $\mathcal{D}$-Lipschitz with $\mathcal{D}$-function $\psi_{\mathcal{A}}$

(b) $\mathcal{B}$ is partially continuous and uniformly partially compact;

(c) $M_{\mathcal{B}} \psi_{\mathcal{A}}(r)<r, r>0$, where $M_{\mathcal{B}}=\sup \{\|\mathcal{B}(C)\|: C$ is a chain in $E\}$;

(d) there exists an element $x_{0} \in X$ such that $x_{0} \preceq \mathcal{A} x_{0} \mathcal{B} x_{0}$ or $x_{0} \succeq \mathcal{A} x_{0} \mathcal{B} x_{0}$.

Then the operator equation

$$
\mathcal{A} x \mathcal{B} x=x
$$

has a positive solution $x^{*}$ in $E$, and the sequence $\left\{x_{n}\right\}$ of successive iterations defined by $x_{n+1}=\mathcal{A} x_{n} \mathcal{B} x_{n}, n=0,1, \ldots$, converges monotonically to $x^{*}$.

Remark 11. The $\mathcal{D}$-compatibility of the order relation $\preceq$ and the norm $\|\cdot\|$ in every compact chain of $E$ holds if every partially compact subset of $E$ 
possesses the $\mathcal{D}$-compatibility property with respect to $\preceq$ and $\|\cdot\|$. This simple fact will be utilized to prove the main results in this paper.

Remark 12. Hypothesis (a) in Theorem 10 implies that the operator $\mathcal{A}$ is partially continuous and consequently both operators in the theorem are partially continuous on $E$. The regularity of $E$ in Theorem 10 may be replaced with a stronger continuity condition on the operators $\mathcal{A}$ and $\mathcal{B}$.

\section{Existence and Approximation Result}

The QHFIE (3) with delay and maxima is considered in the function space $C(J, \mathbb{R})$ of continuous real-valued functions defined on $J$. We define a norm $\|\cdot\|$ and the order relation $\leq$ in $C(J, \mathbb{R})$ by

$$
\|x\|=\sup _{t \in J}|x(t)|
$$

and

$$
x \leq y \text { if and only if } x(t) \leq y(t) \text { for all } t \in J,
$$

respectively.

Clearly, $C(J, \mathbb{R})$ is a Banach space with respect to the above supremum norm and it is partially ordered with respect to the above partially order relation $\leq$. Moreover, $C(J, \mathbb{R})$ is also a Banach algebra with respect to the multiplication ". " defined by

$$
(x \cdot y)(t)=x(t) \cdot y(t) \text { for all } t \in J .
$$

It is known that the partially ordered Banach algebra $C(J, \mathbb{R})$ has some nice properties concerning the $\mathcal{D}$-compatibility property with respect to the norm $\|\cdot\|$ and the order relation $\leq$ in certain of its subsets. The following lemma in this connection follows by an application of the Arzelà-Ascoli theorem.

Lemma 13. Let $(C(J, \mathbb{R}), \leq,\|\cdot\|)$ be a partially ordered Banach space with the norm $\|\cdot\|$ and the order relation $\leq$ defined by (8) and (9), respectively. Then every partially compact subset $S$ of $C(J, \mathbb{R})$ is Janhavi, i.e., $\|\cdot\|$ and $\leq$ are $\mathcal{D}$-compatible in every compact chain $C$ in $S$.

Proof. This lemma is mentioned in Dhage [5, 6], but the proof appears in Dhage $[8,9]$ and Dhage and Dhage $[12,13,14]$. Since the proof is easily available, we omit the details. 
We introduce an order relation $\leq_{\mathcal{C}}$ in $\mathcal{C}$ induced by the order relation $\leq$ defined in $C(J, \mathbb{R})$. This will avoid the confusion of comparison between the elements of the two Banach spaces $\mathcal{C}$ and $C(J, \mathbb{R})$. Thus, for any $x, y \in \mathcal{C}$, $x \leq_{\mathcal{C}} y$ implies $x(\theta) \leq y(\theta)$ for all $\theta \in I_{0}$. Note that if $x, y \in C(J, \mathbb{R})$ and $x \leq y$, then $x_{t} \leq_{\mathcal{C}} y_{t}$ for all $t \in I$.

We need the next definition in what follows.

Definition 14. A function $u \in C(J, \mathbb{R})$ is said to be a lower solution of the QHFIE (1), if it satisfies

$$
\left.\begin{array}{c}
\left(\frac{u(t)}{f(t, u(t), U(t))}\right)^{\prime}+\lambda\left(\frac{u(t)}{f(t, u(t), U(t))}\right) \leq g\left(t, u(t), u_{t}\right), t \in I, \\
u_{0}=\phi \in \mathcal{C},
\end{array}\right\}
$$

where $U(t)=\max _{0 \leq \xi \leq t} u(\xi)$. Similarly, a function $v \in C(J, \mathbb{R})$ is said to be an upper solution of the QHFIE (1) if it satisfies the conditions above with the inequality reversed.

Definition 15. A function $g(t, x, y)$ is called Carathéodory, if

(i) the map $t \mapsto g(t, x, y)$ is measurable for each $x, y \in \mathbb{R}$, and

(ii) the map $(x, y) \mapsto g(t, x, y)$ is jointly continuous for each $t \in J$.

A Caratheódory function $g$ is called $L^{1}$-Carathéodory, if

(iii) there exists a function $h \in L^{1}(I, \mathbb{R})$ such that

$$
|g(t, x, y)| \leq h(t) \text { a.e. } t \in I
$$

for all $x, y \in \mathbb{R}$.

The following lemma is obvious.

Lemma 16. If the function $f(t, x, y)$ is $L^{1}$-Carathéodory, then the function $t \mapsto f(t, x, y)$ is Lebesgue integrable for each $x, y \in \mathbb{R}$.

We will make use of the following assumptions:

$\left(\mathrm{A}_{1}\right) f$ defines a continuous function $f: I \times \mathbb{R} \times \mathbb{R} \rightarrow \mathbb{R}_{+}$. 
$\left(\mathrm{A}_{2}\right)$ The map $x \mapsto \frac{x}{f(0, x, x)}$ is an injection on $\mathbb{R}$.

$\left(\mathrm{A}_{3}\right)$ The function $t \mapsto F(t)=f(t, 0,0$,$) is bounded on J$ with bound $F_{0}$.

$\left(\mathrm{A}_{4}\right)$ There exist constants $L>0$ and $K>0$ such that

$$
0 \leq f\left(t, x_{1}, x_{2}\right)-f\left(t, y_{1}, y_{2}\right) \leq \frac{L \max \left\{x_{1}-y_{1}, x_{2}-y_{2}\right\}}{K+\max \left\{x_{1}-y_{1}, x_{2}-y_{2}\right\}}
$$

for all $t \in I$ and $x_{1}, x_{2}, y_{1}, y_{2} \in \mathbb{R}$ with $x_{1} \geq y_{1}$ and $x_{2} \geq y_{2}$.

(B) $g$ defines a function $g: I \times \mathbb{R} \times \mathcal{C} \rightarrow \mathbb{R}_{+}$.

$\left(\mathrm{B}_{2}\right) g$ is $L^{1}$-Carathéodory.

(B $\left.\mathrm{B}_{3}\right) g(t, x, y)$ is nondecreasing in $x$ and $y$ for all $t \in I$.

$\left(\mathrm{C}_{1}\right)$ The QHFDE (3) has a lower solution $u \in C(J, \mathbb{R})$.

$\left(\mathrm{C}_{2}\right)$ The QHFDE (3) has a upper solution $v \in C(J, \mathbb{R})$.

Remark 17. Note that condition $\left(\mathrm{A}_{2}\right)$ holds if the mapping $x \rightarrow \frac{x}{f(0, x, x)}$ is increasing in $\mathbb{R}$.

The following lemma should be clear.

Lemma 18. Assume that $\left(B_{2}\right)$ and $\left(B_{3}\right)$ hold. Then, a function $x \in$ $C(J, \mathbb{R})$ is a solution of the QHFDE (1) if and only if it is a solution of the integral equation

$$
x(t)=\left\{\begin{array}{l}
{[f(t, x(t), X(t))]\left(c e^{-\lambda t}\right.} \\
\left.\quad+e^{-\lambda t} \int_{0}^{t} g\left(s, x(s), x_{s}\right) d s\right), \quad \text { if } t \in I, \\
\phi(t), \quad \text { if } t \in I_{0},
\end{array}\right.
$$

where $c=\frac{\phi(0)}{f(0, \phi(0), \phi(0))}$. 
Theorem 19. Assume that conditions $\left(A_{1}\right)-\left(A_{4}\right),\left(B_{1}\right)-\left(B_{3}\right)$, and $\left(C_{1}\right)$ hold and

$$
L\left(\left|\frac{\phi(0)}{f(0, \phi(0), \phi(0))}\right|+\|\phi\|+\|h\|_{L^{1}}\right) \leq K .
$$

If $\phi(0) \geq 0$, then the QHFDE (1) has a solution $x^{*}$ defined on $J$, and the sequence $\left\{x_{n}\right\}_{n \in \mathbb{N} \cup\{0\}}$ of successive approximations defined by

$$
\begin{aligned}
& x_{0}=u, \\
& x_{n+1}(t)=\left\{\begin{array}{c}
{\left[f\left(t, x_{n}(t), X_{n}(t)\right)\right]\left(c e^{-\lambda t}\right.} \\
\left.\quad+e^{-\lambda t} \int_{0}^{t} g\left(s, x_{n}(s), x_{s}^{n}\right) d s\right), \quad \text { if } t \in I, \\
\phi(t), \quad \text { if } t \in I_{0},
\end{array}\right.
\end{aligned}
$$

where $x_{s}^{n}=x(s+\theta), \theta \in I_{0}$, converges monotonically to $x^{*}$.

Proof. Set $E=C(J, \mathbb{R})$. Then, in view of Lemma 13, every partially compact subset $S$ of $E$ possesses the $\mathcal{D}$-compatibility property with respect to the norm $\|\cdot\|$ and the order relation $\leq$ so that every compact chain $C$ in $E$ is Janhavi. Define two operators $\mathcal{A}$ and $\mathcal{B}$ on $E$ by

$$
\mathcal{A} x(t)=\left\{\begin{array}{l}
f\left(t, x_{n}(t), X_{n}(t)\right), \quad \text { if } t \in I, \\
1, \quad \text { if } t \in I_{0} .
\end{array}\right.
$$

and

$$
\mathcal{B} x(t)=\left\{\begin{array}{l}
c e^{-\lambda t}+e^{-\lambda t} \int_{0}^{t} e^{\lambda s} g\left(s, x_{n}(s), x_{s}^{n}\right) d s, \quad \text { if } t \in I, \\
\phi(t), \quad \text { if } t \in I_{0} .
\end{array}\right.
$$

From the continuity of the integral, it follows that $\mathcal{A}$ and $\mathcal{B}$ define the operators $\mathcal{A}, \mathcal{B}: E \rightarrow \mathcal{K}$. Applying Lemma 18, the HFDE (11) is equivalent to the operator equation

$$
\mathcal{A} x(t) \mathcal{B} x(t)=x(t), t \in J .
$$

We wish to show that the operators $\mathcal{A}$ and $\mathcal{B}$ satisfy all the conditions of Theorem 10. This will be shown in a series of steps.

Step $\mathbf{I}: \mathcal{A}$ and $\mathcal{B}$ are nondecreasing on $E$. 
Let $x, y \in E$ be such that $x \geq y$. Then $x(t) \geq y(t)$ for all $t \in J$. Since $y$ is continuous on $[a, t]$, there exists a $\xi^{*} \in[a, t]$ such that $y\left(\xi^{*}\right)=\max _{a \leq \xi \leq t} y(\xi)$. By the definition of $\leq$, we have $x\left(\xi^{*}\right) \geq y\left(\xi^{*}\right)$. Consequently,

$$
X(t)=\max _{a \leq \xi \leq t} x(\xi) \geq x\left(\xi^{*}\right) \geq y\left(\xi^{*}\right)=\max _{a \leq \xi \leq t} y(\xi)=Y(t)
$$

for each $t \in J$. Then, by $\left(\mathrm{A}_{2}\right)$, we obtain

$$
\begin{aligned}
\mathcal{A} x(t) & = \begin{cases}f(t, x(t), X(t)), & \text { if } t \in I, \\
1, & \text { if } t \in I_{0},\end{cases} \\
& \geq\left\{\begin{array}{l}
f(t, y(t), Y(t)), \\
1, \quad \text { if } t \in I_{0},
\end{array}\right. \\
& =\mathcal{A} y(t)
\end{aligned}
$$

for all $t \in J$. This shows that the operator $\mathcal{A}$ is nondecreasing on $E$. Again, if $x \geq y$, then by the definition of the order relation $\leq, x_{t} \geq_{\mathcal{C}} y_{t}$ for all $t \in I$. Now, from $\left(B_{3}\right)$,

$$
\begin{aligned}
\mathcal{B} x(t) & =\left\{\begin{array}{l}
c e^{-\lambda t}+e^{-\lambda t} \int_{0}^{t} g\left(s, x(s), x_{s}\right) d s, \quad \text { if } t \in I, \\
\phi(t), \quad \text { if } t \in I_{0},
\end{array}\right. \\
& \geq\left\{\begin{array}{l}
c e^{-\lambda t}+e^{-\lambda t} \int_{0}^{t} g\left(s, y(s), y_{s}\right) d s, \quad \text { if } t \in I, \\
\phi(t), \quad \text { if } t \in I_{0},
\end{array}\right. \\
= & \mathcal{B} y(t)
\end{aligned}
$$

for all $t \in J$. This shows that the operator $\mathcal{B}$ is also nondecreasing on $E$.

Step II: $\mathcal{A}$ is partially bounded and partially $\mathcal{D}$-Lipschitz on $E$.

Let $x \in E$; without loss of generality, we assume that $x \geq 0$. Then by $\left(\mathrm{A}_{2}\right)$,

$$
\begin{aligned}
& |\mathcal{A} x(t)| \leq|f(t, x(t), X(t))-f(t, 0,0)|+|f(t, 0,0)| \\
& \leq \frac{L \max \{x(t), X(t)\}}{K+\max \{x(t), X(t)\}}+F_{0}
\end{aligned}
$$




$$
\begin{aligned}
& \leq \frac{L \max \{|x(t)|,|X(t)|\}}{K+\max \{|x(t)|,|X(t)|\}}+F_{0} \\
& \leq \frac{L\|x\|}{K+\|x\|}+F_{0} \leq L+F_{0}
\end{aligned}
$$

for all $t \in I$. Similarly, if $t \in I_{0}$, then we have $|\mathcal{A}(t)| \leq 1$. Therefore,

$$
|\mathcal{A}(t)| \leq L+F_{0}+1
$$

for all $t \in J$. Taking the supremum over $t$ in the above inequality, we obtain

$$
\|\mathcal{A} x\| \leq L+F_{0}+1
$$

for all $x \in E$. Hence, $\mathcal{A}$ is bounded and consequently a partially bounded operator on $E$.

Next, let $x, y \in E$ be such that $x \geq y$. Then, we have

$$
|x(t)-y(t)| \leq|X(t)-Y(t)|
$$

and that

$$
\begin{aligned}
& |X(t)-Y(t)|=X(t)-Y(t) \\
& =\max _{t_{0} \leq \xi \leq t} x(\xi)-\max _{t_{0} \leq \xi \leq t} y(\xi) \leq \max _{t_{0} \leq \xi \leq t}[x(\xi)-y(\xi)] \\
& =\max _{t_{0} \leq \xi \leq t}|x(\xi)-y(\xi)| \leq\|x-y\|
\end{aligned}
$$

for each $t \in I$. As a result, we have

$$
\begin{aligned}
& |\mathcal{A} x(t)-\mathcal{A} y(t)|=|f(t, x(t), X(t))-f(t, y(t), Y(t))| \\
& \leq \frac{L \max \{|x(t)-y(t)|,|X(t)-Y(t)|\}}{K+\max \{|x(t)-y(t)|,|X(t)-Y(t)|\}} \\
& \leq \frac{L\|x-y\|}{K+\|x-y\|}=\psi_{\mathcal{A}}(\|x-y\|)
\end{aligned}
$$

for all $t \in J$, where $\psi_{\mathcal{A}}(r)=\frac{L r}{K+r}$. Taking the supremum over $t$, we obtain

$$
\|\mathcal{A} x-\mathcal{A} y\| \leq \psi_{\mathcal{A}}(\|x-y\|)
$$

for all $x, y \in E$ with $x \geq y$. Hence, $\mathcal{A}$ is a partial nonlinear $\mathcal{D}$-Lipschitz operator on $E$ with a $\mathcal{D}$-function $\psi_{\mathcal{A}}$, and this further implies that $\mathcal{A}$ is a partially continuous operator on $E$. 
Step III: $\mathcal{B}$ is partially continuous on $E$.

Let $\left\{x_{n}\right\}_{n \in \mathbb{N}}$ be a sequence in a chain $C$ such that $x_{n} \rightarrow x$ as $n \rightarrow \infty$. Then $x_{s}^{n} \rightarrow x_{s}$ as $n \rightarrow \infty$. Since $f$ is continuous, we have

$$
\begin{aligned}
\lim _{n \rightarrow \infty} \mathcal{B} x_{n}(t) & =\left\{\begin{array}{l}
c e^{-\lambda t}+e^{-\lambda t} \int_{0}^{t}\left[\lim _{n \rightarrow \infty} e^{\lambda s} g\left(s, x_{n}(s), x_{s}^{n}\right)\right] d s, t \in I, \\
\phi(t), t \in I_{0}
\end{array}\right. \\
& =\left\{\begin{array}{l}
c e^{-\lambda t}+e^{-\lambda t} \int_{0}^{t} e^{\lambda s} g\left(s, x(s), x_{s}\right) d s, t \in I, \\
\phi(t), t \in I_{0},
\end{array}\right. \\
= & \mathcal{B} x(t)
\end{aligned}
$$

for all $t \in J$. This shows that $\mathcal{B} x_{n}$ converges to $\mathcal{B} x$ pointwise on $J$.

To show that $\left\{\mathcal{B} x_{n}\right\}_{n \in \mathbb{N}}$ is an equicontinuous sequence of functions in $E$, we need to consider three cases.

Case I: Let $t_{1}, t_{2} \in J$ with $t_{1}>t_{2} \geq 0$. Then,

$$
\begin{gathered}
\left|\mathcal{B} x_{n}\left(t_{2}\right)-\mathcal{B} x_{n}\left(t_{1}\right)\right| \\
=\left|e^{t_{2}} \int_{0}^{t_{2}} g\left(s, x_{n}(s), x_{s}^{n}\right) d s-e^{t_{1}} \int_{0}^{t_{1}} g\left(s, x_{n}(s), x_{s}^{n}\right) d s\right|+\left|c e^{\lambda t_{2}}-c e^{\lambda t_{1}}\right| \\
\leq\left|e^{t_{2}} \int_{0}^{t_{2}} g\left(s, x_{n}(s), x_{s}^{n}\right) d s-e^{t_{2}} \int_{0}^{t_{1}} g\left(s, x_{n}(s), x_{s}^{n}\right) d s\right| \\
+\left|e^{t_{2}} \int_{0}^{t_{1}} g\left(s, x_{n}(s), x_{s}^{n}\right) d s-e^{t_{1}} \int_{0}^{t_{1}} g\left(s, x_{n}(s), x_{s}^{n}\right) d s\right|+|c|\left|e^{\lambda t_{2}}-e^{\lambda t_{1}}\right| \\
\leq e^{t_{2}}\left|\int_{0}^{t_{2}} g\left(s, x_{n}(s), x_{s}^{n}\right) d s-\int_{0}^{t_{1}} g\left(s, x_{n}(s), x_{s}^{n}\right) d s\right| \\
\quad+\left|e^{t_{2}}-e^{t_{1}}\right|\left|\int_{0}^{t_{1}} g\left(s, x_{n}(s), x_{s}^{n}\right) d s\right|+|c|\left|e^{\lambda t_{2}}-e^{\lambda t_{1}}\right| \\
\leq e^{t_{2}}\left|\int_{t_{1}}^{t_{2}} g\left(s, x_{n}(s), x_{s}^{n}\right) d s\right| \\
\quad+\left|e^{t_{2}}-e^{t_{1}}\right| \int_{0}^{t_{1}}\left|g\left(s, x_{n}(s), x_{s}^{n}\right)\right| d s+|c|\left|e^{\lambda t_{2}}-e^{\lambda t_{1}}\right|
\end{gathered}
$$




$$
\leq e^{T} M_{\mathcal{B}}\left|t_{2}-t_{1}\right|+\left(|c|+M_{\mathcal{B}} T\right)\left|e^{t_{2}}-e^{t_{1}}\right| \rightarrow 0
$$

as $t_{2} \rightarrow t_{1}$, uniformly for all $n \in \mathbb{N}$.

Case II: Let $t_{1}, t_{2} \in J$ with $t_{1}<t_{2} \leq 0$. Then we have

$$
\left|\mathcal{B} x_{n}\left(t_{2}\right)-\mathcal{B} x_{n}\left(t_{1}\right)\right|=\left|\phi\left(t_{2}\right)-\phi\left(t_{1}\right)\right| \rightarrow 0 \quad \text { as } \quad t_{2} \rightarrow t_{1},
$$

uniformly for all $n \in \mathbb{N}$.

Case III: Let $t_{1}, t_{2} \in J$ with $t_{1}<0<t_{2}$. Then we have

$$
\left|\mathcal{B} x_{n}\left(t_{2}\right)-\mathcal{B} x_{n}\left(t_{1}\right)\right| \leq\left|\mathcal{B} x_{n}\left(t_{2}\right)-\mathcal{B} x_{n}(0)\right|+\left|\mathcal{B} x_{n}(0)-\mathcal{B} x_{n}\left(t_{1}\right)\right| \rightarrow 0
$$

as $t_{2} \rightarrow t_{1}$.

Thus, in all above three cases, we obtain

$$
\left|\mathcal{B} x_{n}\left(t_{2}\right)-\mathcal{B} x_{n}\left(t_{1}\right)\right| \rightarrow 0 \quad \text { as } \quad t_{2} \rightarrow t_{1},
$$

uniformly for all $n \in \mathbb{N}$. This shows that the convergence $\mathcal{B} x_{n} \rightarrow \mathcal{B} x$ is uniform and that $\mathcal{B}$ is a partially continuous operator on $E$ into itself (see Remark 2.1).

Step IV: $\mathcal{B}$ is a partially compact operator on $E$.

Let $C$ be an arbitrary chain in $E$. We will show that $\mathcal{B}(C)$ is a uniformly bounded and equicontinuous set in $E$. First, we show that $\mathcal{B}(C)$ is uniformly bounded. Let $y \in \mathcal{B}(C)$; then there exists $x \in C$ such that $y=\mathcal{B} x$. By $\left(\mathrm{B}_{2}\right)$,

$$
\begin{aligned}
|y(t)| & =|\mathcal{B} x(t)| \\
& \leq\left\{\begin{array}{l}
|c|+e^{-\lambda t} \int_{0}^{t}\left|g\left(s, x(s), x_{s}\right)\right| d s, \quad \text { if } t \in I, \\
|\phi(t)|, \quad \text { if } t \in I_{0} .
\end{array}\right. \\
& \leq|c|+\|\phi\|+\|h\|_{L^{1}}=r
\end{aligned}
$$

for all $t \in J$. Taking the supremum over $t$ we obtain $\|y\| \leq\|\mathcal{B} x\| \leq r$ for all $y \in \mathcal{B}(C)$. Hence, $\mathcal{B}(C)$ is a uniformly bounded subset of $E$.

Next we show that $\mathcal{B}(C)$ is an equicontinuous set in $E$. Let $t_{1}, t_{2} \in J$, with $t_{1}<t_{2}$. Then proceeding with arguments similar to those used in Step II, it can be shown that

$$
\left|y\left(t_{2}\right)-y\left(t_{1}\right)\right|=\left|\mathcal{B} x\left(t_{2}\right)-\mathcal{B} x\left(t_{1}\right)\right| \rightarrow 0 \quad \text { as } \quad t_{1} \rightarrow t_{2}
$$

uniformly for all $y \in \mathcal{B}(C)$. This shows that $\mathcal{B}(C)$ is an equicontinuous subset of $E$. Now, $\mathcal{B}(C)$ is a uniformly bounded and equicontinuous subset of functions 
in $E$, and hence it is compact in view of Arzelà-Ascoli theorem. Consequently, $\mathcal{B}: E \rightarrow E$ is a partially compact operator on $E$ into itself.

Step V: $u$ is a lower solution of the operator equation (16).

By condition $\left(\mathrm{C}_{1}\right)$, the HFDE (3) has a lower solution $u$ defined on $J$. Then we have

$$
\left.\begin{array}{c}
\left(\frac{u(t)}{f(t, u(t), U(t))}\right)^{\prime}+\lambda\left(\frac{u(t)}{f(t, u(t), U(t))}\right) \leq g\left(t, u(t), u_{t}\right), t \in I, \\
u_{0}=\phi \in \mathcal{C},
\end{array}\right\}
$$

where $U(t)=\max _{0 \leq \xi \leq t} u(\xi)$. Integrating the above inequality from 0 to $t$ gives

$$
\begin{aligned}
u(t) & \leq\left\{\begin{array}{l}
{[f(t, u(t), U(t))]\left(c e^{-\lambda t}+e^{-\lambda t} \int_{0}^{t} g\left(s, u(s), u_{s}\right) d s\right), t \in I,} \\
\phi(t), t \in I_{0}
\end{array}\right. \\
& =\mathcal{A} u(t) \mathcal{B} u(t)
\end{aligned}
$$

for all $t \in J$. As a result we have $u \leq \mathcal{A} u \mathcal{B} u$ and so $u$ is a lower solution of the operator equation (16) defined on $J$.

Finally, by condition (12), we obtain

$$
M_{\mathcal{B}} \psi_{\mathcal{A}}(r) \leq\left(\left|\frac{\phi(0)}{f(0, \phi(0), \phi(0))}\right|+\|\phi\|+\|h\|_{L^{1}}\right) \cdot \frac{L r}{K+r}<r
$$

for each $r>0$, and so condition (c) of Theorem 10 is satisfied.

Thus, the operators $\mathcal{A}$ and $\mathcal{B}$ satisfy all the conditions of Theorem 10 and so the operator equation $\mathcal{A} x \mathcal{B} x=x$ has a positive solution $x^{*}$. Consequently, the integral equation (11), and a fortiori the QHFDE (3), has a positive solution $x^{*}$ defined on $J$. Furthermore, the sequence $\left\{x_{n}\right\}_{n=0}^{\infty}$ of successive approximations defined by (12) converges monotonically to $x^{*}$. This completes the proof of the theorem.

Remark 20. The conclusion of Theorems 19 also remains true if we replace condition $\left(\mathrm{C}_{1}\right)$ by $\left(\mathrm{C}_{2}\right)$.

Example 21. Given two closed and bounded intervals $I_{0}=\left[-\frac{\pi}{2}, 0\right]$ and $I=[0,1]$ of the real line $\mathbb{R}$ and an initial function $\phi \in \mathcal{C}$, consider the QHFDE 
with a delay and maxima

$$
\left.\begin{array}{c}
\left(\frac{x(t)}{f_{1}(t, x(t), X(t))}\right)^{\prime}+\lambda\left(\frac{x(t)}{f_{1}(t, x(t), X(t))}\right)=g_{1}\left(t, x(t), x_{t}\right), t \in I, \\
x_{0}=\phi
\end{array}\right\}
$$

where $\lambda \in \mathbb{R}, \lambda>0, X(t)=\max _{0 \leq \xi \leq t} x(\xi), J=\left[-\frac{\pi}{2}, 1\right]$, and $f_{1}: I \times \mathbb{R} \times \mathbb{R} \rightarrow \mathbb{R} \backslash\{0\}$ and $g_{1}: I \times \mathbb{R} \times \mathcal{C} \rightarrow \mathbb{R}$ are continuous functions given by

$$
\begin{aligned}
\phi(\theta) & =\sin \theta, \quad \theta \in\left[-\frac{\pi}{2}, 0\right] \\
f_{1}(t, x, y) & = \begin{cases}\frac{x+y}{1+x+y}+1, & \text { if } x>0, y>0 \\
1, & \text { if } x \leq 0, y \leq 0\end{cases}
\end{aligned}
$$

and

$$
g_{1}(t, x, y)=\left\{\begin{aligned}
\frac{1}{3}\left[\tanh x+\tanh \left(\|y\|_{\mathcal{C}}\right)+1\right], & \text { if } x>0, y \geq_{\mathcal{C}} 0, y \neq 0, \\
\frac{1}{3}, & \text { if } x \leq 0, y \leq_{\mathcal{C}} 0,
\end{aligned}\right.
$$

for all $t \in I$. We will show that the functions $f_{1}$ and $g_{1}$ satisfies all the hypotheses of Theorem 19. Clearly, $f_{1}$ is a continuous and positive function on $[0,1] \times \mathbb{R} \times \mathbb{R}$ and so $\left(\mathrm{A}_{1}\right)$ is satisfied. To show that the map $x \rightarrow \frac{x}{f(0, x, x)}$ is an injection on $\mathbb{R}$, let $x>0$ and $y>0$ be any two real numbers. Then the expression

$$
\frac{x}{f(0, x, x)}=\frac{y}{f(0, y, y)}
$$

implies that

$$
\frac{x}{2 x /(1+2 x)}=\frac{y}{2 y /(1+2 y)} \text { so } x=y \text {. }
$$

Similarly, if $x \leq 0$ and $y \leq 0$,

$$
\frac{x}{f(0, x, x)}=\frac{y}{f(0, y, y)} \text { implies } x=y \text {. }
$$

This proves that $x \rightarrow \frac{x}{f(0, x, x)}$ is injective on $\mathbb{R}$, and so $\left(\mathrm{A}_{2}\right)$ is satisfied. 
We have here $F(t)=f_{1}(t, 0,0)=1$ for all $t \in[0,1]$, and so $\left(\mathrm{A}_{3}\right)$ holds. To show $\left(\mathrm{A}_{4}\right)$ holds, let $\left(x_{1}, y_{1}\right),\left(x_{2}, y_{2}\right) \in \mathbb{R} \times \mathbb{R}$ be such that $x_{1} \geq y_{1}$ and $x_{2} \geq y_{2}$. Then, by the definition of $f_{1}$,

$$
\begin{aligned}
0 & \leq f\left(t, x_{1}, y_{1}\right)-f\left(t, x_{2}, y_{2}\right) \\
& =\frac{1}{4} \cdot\left[\frac{x_{1}+x_{2}}{1+x_{1}+x_{2}}-\frac{y_{1}+y_{2}}{1+y_{1}+y_{2}}\right] \\
& \leq \frac{1}{4} \cdot \frac{x_{1}-y_{1}+x_{2}-y_{2}}{\left(1+x_{1}+x_{2}\right)\left(1+y_{1}+y_{2}\right)} \\
& \leq \frac{1}{4} \cdot \frac{x_{1}-y_{1}+x_{2}-y_{2}}{1+x_{1}+x_{2}+y_{1}+y_{2}} \\
& \leq \frac{1}{4} \cdot \frac{x_{1}-y_{1}+x_{2}-y_{2}}{1+x_{1}-y_{1}+x_{2}-y_{2}} \\
& \leq \frac{1}{4} \cdot \frac{2 \max \left\{x_{1}-y_{1}, x_{2}-y_{2}\right\}}{1+\max \left\{x_{1}-y_{1}, x_{2}-y_{2}\right\}} \\
& =\frac{1}{2} \cdot \frac{\max \left\{x_{1}-y_{1}, x_{2}-y_{2}\right\}}{1+\max \left\{x_{1}-y_{1}, x_{2}-y_{2}\right\}},
\end{aligned}
$$

and so $\left(\mathrm{A}_{4}\right)$ holds with $L=\frac{1}{2}$ and $K=1$.

Now the function $g_{1}$ is continuous and positive on $I \times \mathbb{R} \times \mathcal{C}$, and with $h(t)=1$ for all $t \in I, x \in \mathbb{R}$, and $y \in \mathcal{C}$, we see that $g_{1}$ is an $L^{1}$-Carathéodory function on $I \times \mathbb{R} \times \mathcal{C}$. Thus, $\left(\mathrm{B}_{1}\right)$ and $\left(\mathrm{B}_{2}\right)$ are satisfied. Also the function

$$
g_{1}(t, x, y)=\frac{1}{3}\left[\tanh x+\tanh \left(\|y\|_{\mathcal{C}}\right)+1\right]
$$

is nondecreasing in $x$ and $y$ for each $t \in I$, so $\left(\mathrm{B}_{3}\right)$ holds. Furthermore, here $M_{\mathcal{B}} \leq|c|+\|\phi\|+\|h\|_{L^{1}}=2$. Therefore, we have

$$
M_{\mathcal{B}} \psi_{A}(r) \leq 2 \cdot \frac{1}{2} \cdot \frac{r}{1+r}<r
$$

for each $r>0$. Finally, it can be shown that the function

$$
u(t)= \begin{cases}\frac{1}{3} t e^{-\lambda t}, & \text { if } t \in[0,1], \\ \sin t, & \text { if } t \in\left[-\frac{\pi}{2}, 0\right],\end{cases}
$$


is a lower solution of the QHFDE (17) defined on $J$. Thus, all the conditions of Theorem 19 are satisfied. Hence, the QHFDE (17) has a positive solution $x^{*}$ and the sequence $\left\{x_{n}\right\}$ of successive approximations defined by

$$
\begin{aligned}
x_{0}= \begin{cases}\frac{1}{3} t e^{-\lambda t} t, & \text { if } t \in[0,1], \\
\sin t, & \text { if } t \in\left[-\frac{\pi}{2}, 0\right],\end{cases} \\
x_{n+1}(t)=\left\{\begin{array}{l}
{\left[f_{1}\left(t, x_{n}(t), X_{n}(t)\right)\right]\left(c e^{-\lambda t}\right.} \\
\left.+e^{-\lambda t} \int_{0}^{t} g_{1}\left(s, x_{n}(s), x_{s}^{n}\right) d s\right), \\
\left.\sin t, \quad \text { if } t \in\left[-\frac{\pi}{2}, 0\right], 1\right],
\end{array}\right.
\end{aligned}
$$

where $x_{s}^{n}=x(s+\theta), \theta \in I_{0}$, converges monotonically to $x^{*}$.

Remark 22. The conclusion obtained in Example 3.1 also remains true if we replace the lower solution $u$ by the upper solution $v$ of the QHFDE (17) given by

$$
v(t)= \begin{cases}2 t e^{-\lambda t}, & \text { if } t \in[0,1] \\ \sin t, & \text { if } t \in\left[-\frac{\pi}{2}, 0\right]\end{cases}
$$

Remark 23. We note that the special case of the QHFDE (3) in the form

$$
\left.\begin{array}{c}
\left(\frac{x(t)}{f(t, x(t))}\right)^{\prime}+\lambda\left(\frac{x(t)}{f(t, x(t))}\right)=g\left(t, x_{t}\right), t \in I, \\
x_{0}=\phi \in \mathcal{C} .
\end{array}\right\}
$$

has been considered in Mule and Ahirrao [24], but the proof of their main existence theorem is not correct, and in fact is a duplication of the proof of the existence and approximation theorem for the FDE

$$
\left.\begin{array}{rl}
\left(\frac{x(t)}{f(t, x(t))}\right)^{\prime} & =g\left(t, x_{t}\right), \quad t \in I, \\
x_{0} & =\phi \in \mathcal{C} .
\end{array}\right\}
$$

given in Dhage and Dhage [14] and Dhage [11]. The QHFDE (19) has also been studied in Dhage et. al [20] but only for existence of solutions; no information 
on the positivity or approximation of solutions is obtained. Therefore, our main existence theorem, Theorem 19 includes the existence and approximation theorems for the QHFDEs (18) and (19) which are also new to the literature.

In conclusion, we mention that our existence and approximation theorem, Theorem 19 above, may be extended with appropriate modifications to the QHFDE of neutral type

$$
\left.\begin{array}{c}
\left(\frac{x(t)}{f\left(t, x(t), x_{t}\right)}\right)^{\prime}+\lambda\left(\frac{x(t)}{f\left(t, x(t), x_{t}\right)}\right)=g\left(t, x(t), x_{t}\right), t \in I, \\
x_{0}=\phi \in \mathcal{C},
\end{array}\right\}
$$

where $\lambda \in \mathbb{R}, \lambda>0, f: I \times \mathbb{R} \times \mathcal{C} \rightarrow \mathbb{R} \backslash\{0\}, g: I \times \mathbb{R} \times \mathcal{C} \rightarrow \mathbb{R}$ are continuous functions.

\section{References}

[1] S. Chandrasekher, Radiative Transfer, Dover Publications, New York (1960).

[2] K. Deimling, Nonlinear Functional Analysis, Springer-Verlag, Berlin (1985).

[3] B.C. Dhage, Quadratic perturbations of periodic boundary value problems of second order ordinary differential equations, Differ. Equ. Appl., 2 (2010), $465-486$.

[4] B.C. Dhage, Fixed point theorems in ordered Banach algebras and applications, PanAmer. Math. J., 9, No 4 (1999), 93-102.

[5] B.C. Dhage, Hybrid fixed point theory in partially ordered normed linear spaces and applications to fractional integral equations, Differ. Equ. Appl., 5 (2013), 155-184.

[6] B.C. Dhage, Partially condensing mappings in partially ordered normed linear spaces and applications to functional integral equations, Tamkang J. Math., 45, No 4 (2014), 397-427.

[7] B.C. Dhage, Nonlinear $\mathcal{D}$-set-contraction mappings in partially ordered normed linear spaces and applications to functional hybrid integral equations, Malays. J. Mat. Sci., 3, No 1 (2015), 62-85. 
[8] B.C. Dhage, Some generalizations of a hybrid fixed point theorem in a partially ordered metric space and nonlinear functional integral equations, Differ. Equ. Appl., 8 (2016), 77-97.

[9] B.C. Dhage, Approximating solutions of nonlinear periodic boundary value problems with maxima, Cogent Mathematics, 3 (2016), \# 1206699.

[10] B.C. Dhage, Dhage iteration method for nonlinear first order ordinary hybrid differential equations with mixed perturbation of second type with maxima, J. Nonlinear Funct. Anal., 2016 (2016), Article ID 31.

[11] B.C. Dhage, Dhage iteration method in the theory of initial value problems of nonlinear first order hybrid differential equations, Malays. J. Mat. Sci., 5, No 4 (2017), to appear.

[12] B.C. Dhage and S.B. Dhage, Approximating solutions of nonlinear pbvps of hybrid differential equations via hybrid fixed point theory, Indian $J$. Math., 57, No 1 (2015), 103-119.

[13] B.C. Dhage and S.B. Dhage, Approximating solutions of nonlinear first order ordinary differential equations, GJMS Special Issue for Recent Advances in Mathematical Sciences and Applications - 13 (GJMS), 2, No 2 (2013), 25-35.

[14] B.C. Dhage and S.B. Dhage, Approximating positive solutions of nonlinear first order ordinary hybrid differential equations, Cogent Mathematics, 2 (2015), \# 1023671.

[15] B.C. Dhage and S.B. Dhage, Approximating positive solutions of PBVPs of nonlinear first order ordinary hybrid differential equations, Appl. Math. Lett., 46 (2015), 133-142.

[16] S.B. Dhage and B.C. Dhage, Dhage iteration method for approximating positive solutions of PBVPs of nonlinear hybrid differential equations with maxima, Intern. Jour. Anal. Appl., 10, No 2 (2016), 101-111.

[17] S.B. Dhage and B.C. Dhage, Dhage iteration method for approximating positive solutions of nonlinear first order ordinary quadratic differential equations with maxima, Nonlinear Anal. Forum, 16, No 1 (2016), 87-100.

[18] B.C. Dhage, S.B. Dhage, and J.R. Graef, Dhage iteration method for initial value problems for nonlinear first order hybrid integro-differential equations, J. Fixed Point Theory Appl., 18 (2016), 309-326. 
[19] B.C. Dhage, S.B. Dhage, and J.R. Graef, Dhage iteration method for nonlinear first order hybrid differential equations with a mixed perturbation of the second type, Dyn. Contin. Discrete Impuls. Syst., Ser. A Math. Anal., 24 (2017), 159-180.

[20] B.C. Dhage, N.S. Jadhav, and A.Y. Shete, Hybrid fixed point theorem with PPF dependence in Banach algebras and applications to quadratic differential equations, J. Math. Comput. Sci., 5 (2015), 601-614.

[21] J.K. Hale, Theory of Functional Differential Equations, Springer-Verlag, New York-Berlin (1977).

[22] S. Heikkilä and V. Lakshmikantham, Monotone Iterative Techniques for Discontinuous Nonlinear Differential Equations, Dekker, New York (1994).

[23] A.R. Magomedov, On some questions about differential equations with maxima, Izv. Akad. Nauk Azerbaidzhan. SSR Ser. Fiz.-Tehn. Mat. Nauk, 1 (1977), 104-108 (in Russian).

[24] D.V. Mule and B.R. Ahirrao, Approximating solution of an initial and periodic boundary value problems for first order quadratic functional differential equations, Int. J. Pure Appl. Math., 113, No 2 (2017), 251-271.

[25] A.D. Myshkis, On some problems of the theory of differential equations with deviating argument, Russian Math. Surveys, 32 (1977), 181-210. 
